\title{
Where is the Evidence? A Critical Review of Bias in the Reporting of Clinical Data for Exparel: A Liposomal Bupivacaine Formulation
}

Rita M. Hadley ${ }^{1}$ and Alan P Dine ${ }^{2^{*}}$

${ }^{1}$ Advanced Surgical Partners, Springboro, $\mathrm{OH}$, USA

${ }^{2}$ BSN, Kimberly Clark Corporation, Pleasant Plain, OH, USA

*Corresponding author: Alan P Dine, BSN, Kimberly Clark Corporation, Pleasant Plain, OH United States, Tel: 513-253-8033; Fax: 513-672-0181; E-mail: Alan.dine@yahoo.com

Rec date: Apr 16, 2014, Acc date: Jul 26, 2014, Pub date: Jul 28, 2014

Copyright: (c) 2014 Hadley RM, et al. This is an open-access article distributed under the terms of the Creative Commons Attribution License, which permits unrestricted use, distribution, and reproduction in any medium, provided the original author and source are credited.

\begin{abstract}
Healthcare policies are formed through the dissemination of clinical information or evidence of effectiveness, so that this evidence can reach clinical practice. Manipulation or 'influence' over the data and the subsequent reporting of data can be introduced by anyone involved in a study. This may affect the perception of the evidence that becomes the basis for the determination of best practice in the care for our patients so it must be free of bias. This report illustrates an example of bias introduction into the clinical evidence portfolio of the recently introduced liposomal encapsulated bupivacaine Exparel ${ }^{\mathrm{TM}}$. This review then uses this new drug as an example of how bias is introduced into the clinical decision tree and its potential effect on the practice of medicine.
\end{abstract}

Keywords: Bias in Clinical Trials, Evidence in Medicine

\section{Introduction}

Today's healthcare policies are formed through the dissemination of clinical information or evidence of effectiveness, so that this evidence can reach clinical practice. Evidence-Based Medicine has been defined as "the conscientious, explicit and judicious use of current best evidence in making decisions about the care of the individual patient" [1]. More often than not, these evidence based principals of best practice are driven by the medical literature and subject to individual interpretation. Physicians are in the unique position to prove what medical decisions are in the patient's best interest and the best way for them to accomplish this is to utilize outcomes data to determine the best treatment for every patient's problem. Ultimately, collecting these data may improve the quality of care.

The aim of clinical trials is to generate new knowledge on the effectiveness of healthcare interventions, whether they are therapeutic or diagnostic in nature. In order to accommodate the need for welldesigned clinical trials the FDA set up regulations and guidelines for the proper conduct of these clinical trials and directed at limiting the potential for bias in studies. It is well understood and accepted that almost all studies have some bias. Whether or not that bias creates fatal flaws resulting in inconclusive evidence depends on the severity and type of bias interjected into the study. At the basis for bias is the reality that researchers typically want a study to show positive outcomes, and their influence or bias generated by various inputs can only be reduced by proper study design and execution from the onset. The critical question in most studies is whether or not the design, execution or interpretation of results could be due in large part to bias of the researchers, thus making all conclusions invalid. For instance, an observational study, e.g., case series, that records certain outcomes as measured by the researchers, is inherently more susceptible to bias than is a strict experimental study design which uses random chance and a control comparator. The choice of the comparator may also add to the risk of injecting bias into the outcomes of a study. It is the control of bias that ultimately leads to flawless creation of evidence, evidence that becomes the basis for the determination of best practice in the care for our patients.

\section{Bias in Clinical Trials}

At its basics, bias could well be defined as a one-sided predisposition of the mind. In clinical trial design, bias (also known as systematic error) is any process or effect that produces results or conclusions that differ from the truth or that may compromise the ability to draw valid conclusions from the clinical data. The far reaching principles of clinical trial design are specifically aimed at minimizing known or suspected sources of bias.

Manipulation or 'influence' over data or situations (the interjection of an opinion, feeling or influence that favors one treatment over another) can be conscious or unconscious and can be introduced by anyone involved in a study. Common types of bias include treatment bias, the act of 'bending' the data in favor of a particular treatment group; selection bias or the assigning of patients to groups in an unbalanced manner, design bias, where trials are designed to get an answer ('Yes' OR 'No'), but they can be designed in a such a way to favor a particular treatment group. Another form of bias is called placebo effect. The human experience associated with taking an action to fix a problem allows the brain to assume that because an action was taken, that action will produce good results. The brain can also believe that because a product is 'new', that it is better and should work, and therefore it does. This effect is very real and can influence data.

When you are assessing the quality of evidence, it's not how the data are analyzed that is important. Far more important is how the data are collected. For example, statisticians may agonize over whether the researchers should have used a non-parametric test or whether a random effects meta-analysis is appropriate (just to cite two obscure examples) or as is noted in this paper the use of cumulative analysis in pain management. These are important issues and they generate a lot 
of debate as to the best statistical test to use in a particular type of study. But in most cases, the use of one statistical analysis or another is unlikely to make a substantial difference in the conclusions of a clinical study. It is the data that are important, how they were collected and the bias injected into that methodology and the reporting of the data is the chief determinant of the impact on the practice of medicine.

\section{Exparel ${ }^{\circledast}$ Review}

An example of flawed data reporting and bias in clinical trials can be found in the evidence reporting the outcomes of the new drug Exparel $^{\circledR}$ (Bupivacaine Liposome Injectable Suspension, Pacira Pharmaceuticals, San Diego, CA). Exparel is an extended release liposome injection of bupivacaine designed to achieve long-acting postoperative analgesia. The drug consists of microscopic, spherical, lipid-based particles (the DepoFoam drug delivery system) composed of a honeycomb of numerous, nonconcentric, internal aqueous chambers containing the encapsulated bupivacaine. Each chamber is separated from adjacent chambers by lipid membranes. This product was originally named Skye 0402 and then following transition of ownership of the product by Pacira was termed generically as DepoFoam ${ }^{\oplus}$ bupivacaine and ultimately Exparel.

The clinical findings reported in the literature for Exparel seemingly allow the manufacture to make claims as a powerful new modality in improving patient care, and also suggest it as a new means for improving economic and operational efficiencies in patient care. However a review of the evidence for Exparel illustrates how various forms of bias in the design, conduct, and analysis associated with the studies performed on the drug can lead to inaccuracies in reporting and potentially mislead the medical community.

The Food and Drug Administration of the United States (FDA) approved Exparel after a review of twenty-two studies including three Phase III studies which focused on hemorrhoidectomy and bunionectomy procedures. Two of the three Phase III studies demonstrated a significant effect over placebo (saline) for the primary outcome of cumulative pain scores over a period limited to less than 24 hours, as noted in the description of the results by the FDA medical reviewer. In the bunionectomy study, the FDA concluded that, "... Exparel demonstrated a significant reduction in pain intensity compared to placebo for up to 24 hours. The difference in mean pain intensity between treatment groups occurred only during the first 24 hours following study drug administration. Between 24 and 72 hours after study drug administration, there was minimal to no difference between Exparel and placebo treatments on mean pain intensity". For the Hemorrhoid study, "Exparel demonstrated a significant reduction in pain intensity compared to placebo for up to 24 hours. The difference in mean pain intensity between treatment groups occurred only during the first 24 hours following study drug administration. Between 24 and 72 hours after study drug administration, there was minimal to no difference between Exparel and placebo treatments on mean pain intensity; however, there was an attendant decrease in opioid consumption, the clinical benefit of which was not demonstrated" $[2,3]$. As illustrated in the discussion of these studies below, the opioid reducing benefit was realized only in the first 12 hours post drug administration (Figure 1). This lack of efficacy beyond 12 hours is apparent in the results that are illustrated in medical and statistical review by the FDA. In a third Phase III study submitted to the FDA as part of the new drug application, a 204-patient hemorrhoidectomy study conducted vs. bupivacaine, none of the 60 endpoints reviewed illustrated a beneficial effect over a single injection of unencapsulated bupivacaine. In fact, in this study a single injection of plain bupivacaine illustrated better results than Exparel. It is of interest as well that this study has never been published outside the medical review by the FDA.

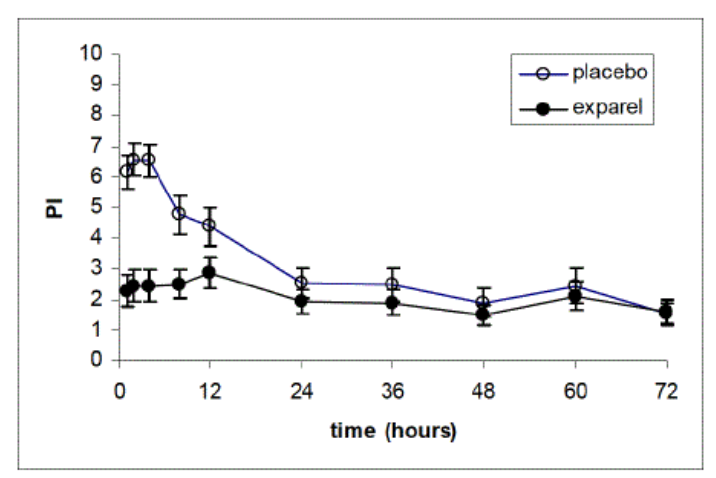

Figure 1: Mean Pain Intensity versus Time plot for hemorrhoidectomy study (C-316) *FDA Amended Clinical Review, Center for Drug Evaluation and Research, October 2011

The FDA medical reviewer for this product further summarized the effect of Exparel in the Clinical Review dated October 7, 2011. In his review he stated; "In the placebo controlled studies, Exparel was significantly better than placebo for reducing pain intensity during the first 12 hours following administration. This effect diminished over the next 12 hours such that by 24 hours after administration there was no clinically relevant difference in the pain experienced by subjects treated with Exparel compared to those treated with normal saline. Based on the demonstration of Exparel's efficacy versus placebo, its pharmacodynamics being similar to that of unencapsulated bupivacaine $\mathrm{HCl}$, the benefits were considered to outweigh the risks. This finding however is limited to the two surgical procedures studied in the placebo-controlled pivotal studies. The manner in which Exparel was administered and the doses used in those studies were so dissimilar that it is not possible to extrapolate a dose or method of administration that would be efficacious for other surgical procedures. To resolve this issue, additional adequate and well-controlled studies would be needed." It is unfortunate that these studies have not been performed as the current evidence for this drug does not reveal any benefit over the currently marketed unencapsulated formulations of bupivacaine.

The pharmacodynamics section of the FDA review reveals the details of the onset and duration of activity of this drug in an explicit manner often overlooked in the clinical manuscripts published on this drug. This report highlights that, "The onset of action of Exparel was evaluated in clinical trials that assessed pain intensity and other outcomes. These studies demonstrated that the onset of action for Exparel was less than 2 minutes, and was similar to conventional bupivacaine $\mathrm{HCl}$. In the clinical trials described in the medical review, the duration of Exparel's analgesic effect appears to be no more than 24 hours and not longer than that of unencapsulated bupivacaine $\mathrm{HCl}$ " [4].

At first glance, the placebo-controlled hemorrhoid study appears to have demonstrated the superior efficacy of Exparel over saline injections in the publication by Gorfine et al. [5] suggesting that the primary endpoint for the hemorrhoidectomy study, AUC0-72 for NRS 
of pain intensity, is superior for the entire 72 hours. This is not the case. As described in the Medical Review section 6.1.9, the analgesia derived from Exparel does not differ from placebo, at least in a clinically meaningful way, beyond 24 hours."

Subsequent to the review and approval of Exparel, adequate and well controlled studies have not been performed. It is the intent of this paper to illustrate the inadequacies and the lack of adherence to good clinical design and reporting that have plagued the efforts of scientific dissemination of information about this product since its approval.

How is it possible that the messages delivered in peer reviewed journals are permitted to be published without question? It is a perfect illustration in bias in reporting in the medical literature and ultimately an injustice to the to the entire medical publication peer review system. In order to illustrate this form of bias, a review of the published studies for this drug is necessary.

Many of the studies conducted with Exparel use a cumulative outcomes analysis (either cumulative opioid use or cumulative pain scores). Cumulative data is used to distract and disguise the truth. The use of discontinuous data permits the interpretation of the effects of a drug or device at specific time points. This not the case with continuous analyses. In the case of the study by Gorfine, the statistics reported out in the paper would not have shown a meaningful difference between the use of Exparel and placebo after 12 hours. Without the use of discrete data, the reader is distracted from this point by assuming that an initial measurable difference was sustained throughout the entire period of study. In order to make a strong statement about the efficacy of a product these discrete data points need to illustrate significant benefits. The need to summarize data with continuous analyses suggests weak associations, at best. The difficulty in interpreting continuous data in this fashion is that the analysis masks what happens at each discrete time-point. Table 1 is taken from the manuscript authored by Gorfine [4] and provides the analysis of opioid rescue medications in a cumulative fashion. By breaking out the data into 12 hour time-points rather than in a cumulative manner the actual difference between Exparel and placebo (saline) is limited to the first 12 hours (Table 1).

\begin{tabular}{|l|l|l|}
\hline Time from $\mathbf{0}$ through: & $\begin{array}{l}\text { Depofoam bupivacaine } \\
\mathbf{3 0 0} \mathbf{~ m g} \\
\mathbf{N = 9 4}\end{array}$ & $\begin{array}{l}\text { Placebo } \\
\mathbf{N}=93\end{array}$ \\
\hline $12 \mathrm{~h}$ postdose & $6.2(8.2) \mathrm{a}$ & $14.7(10.7)$ \\
\hline $24 \mathrm{~h}$ postdose & $11.3(11.8) \mathrm{a}$ & $20.0(13.5)$ \\
\hline $36 \mathrm{~h}$ postdose & $15.7(15.7) \mathrm{a}$ & $23.3(15.9)$ \\
\hline $48 \mathrm{~h}$ postdose & $17.2(17.2) \mathrm{a}$ & $25.4(17.7)$ \\
\hline $60 \mathrm{~h}$ postdose & $21.2(20.2) \mathrm{b}$ & $28.2(20.3)$ \\
\hline $72 \mathrm{~h}$ postdose & $22.3(21.0) \mathrm{c}$ & $29.1(20.7)$ \\
\hline
\end{tabular}

Table 1: Mean (SD) total amount of opioid rescue medication (morphine equivalents) consumed (full analysis set) (Adapted from Gorfine et al.). ${ }^{*} \mathrm{ap}<0.001 \mathrm{bp}<0.0003 \mathrm{cp}<0.0006$

As you can see from these examples, while there was a significant difference noted at the $0-12 \mathrm{~h}$ time point, the differences at each timepoint after that were not statistically significant nor were they clinically relevant and from the 12 hour time point through 72 hours the results favoured the placebo therapy numerically. The authors of this study would lead you to believe that the use of opioid rescue medication was significantly reduced throughout the 72 hour time frame when in actuality a significant effect was illustrated only in the first 12 hours, following that the two therapies are virtually equivalent (Table 2).

\section{Analysis of Gorfine Data by 12 hour Time-Points}

In Table 2 below, the amount of opioid rescue is further described in a non-cumulative manner. The amount of opioid taken during each 12 hour time period is illustrated. Cumulative analysis from time zero can be confusing as it masks the results after 12 hours. Total opioid consumption from 12-72 hours is included in this table to illustrate the lack of difference over that time period.

\begin{tabular}{|l|l|l|}
\hline Time $\mathbf{0 - 7 2} \mathbf{~}$ & $\begin{array}{l}\text { DepoFoam bupivacaine } \\
\mathbf{3 0 0} \mathbf{~ m g}(\mathbf{n}=\mathbf{9 4})\end{array}$ & $\begin{array}{l}\text { Placebo } \\
(\mathbf{n}=\mathbf{9 3})\end{array}$ \\
\hline $0-12 \mathrm{~h}$ postdose & $6.2 \mathrm{a}$ & 14.7 \\
\hline $12-24 \mathrm{~h}$ postdose & 5.1 & 5.3 \\
\hline $24-36 \mathrm{~h}$ postdose & 4.4 & 3.3 \\
\hline $36-48 \mathrm{~h}$ postdose & 1.5 & 2.1 \\
\hline $48-60 \mathrm{~h}$ postdose & 4 & 2.8 \\
\hline $60-72 \mathrm{~h}$ postdose & 1.1 & 0.9 \\
\hline $12-72 \mathrm{~h}$ postdose & 16.1 & 14.4 \\
\hline
\end{tabular}

Table 2: Total opioid consumption from 12-72 hours. ${ }^{*}$ ap $<0.001$

The results of the pivotal bunionectomy study are also plagued by reporting bias. The clinical and statistical review performed by the FDA is quite different from that reported by Golf [6]. The authors of the manuscript concluded that Exparel provided extended pain relief and decreased opioid use after bunionectomy, compared with placebo. The FDA states that, "By 8 hours after study drug administration, both treatment groups have mean scores that are indicative of moderate levels of pain, and are indistinguishable from each other....By 12 hours the pain scores in the Exparel group are as if they had not received any treatment at all." Furthermore the FDA reviewer stated, "Exparel did not seem effective at controlling post-operative pain associated with bunionectomy" [7].

The proliferation of bias in the post market (Phase IV) studies is the cause of the greatest concern. Various forms of bias in these studies bring doubt that this formulation of bupivacaine brings value to the medical community. Two studies in abdominal surgical procedures illustrate these concerns [8,9]. The biases evident in these studies include design flaws, investigator manipulation and faulty reporting.

In both studies, the two groups were not treated equally. In the Cohen study a sequential enrollment schema with assignments made at the discretion of the principle investigator was utilized. The Exparel group was treated with a multi-modal analgesic regimen including IV acetaminophen and IV ibuprofen plus IV and oral opioids. The bupivacaine group was treated with IV and oral opioids only for breakthrough pain. The principle investigator who is a paid consultant for Pacira Pharmaceuticals made all treatment decisions, assignment of study groups and determination of outcomes. By not having independent criteria for discharge, the length of hospital stay was determined by the investigator who was aware of the treatment assignments. Table 1 in the paper lists readmission to the hospital as 
higher in the Exparel group. These costs were not factored into overall costs thus adding bias to the economic analysis. Without the inclusion of the costs of readmission, an economic analysis of the total cost of care cannot be made.

\section{The design of the Marcet study was much like the Cohen study reviewed above.}

The Exparel multi-modal group was treated with $30 \mathrm{mg}$ ketorolac IV (or alternative NSAID equivalent) at the end of surgery, followed by $1000 \mathrm{mg}$ acetaminophen (IV or oral) every 6 hours for 72 hours postsurgery, as well as oral ibuprofen 600mg every 6 hours for 72 hours, and rescue therapy with IV opioid and/or oxycodone/acetaminophen $5 \mathrm{mg} / 325 \mathrm{mg}$. The control group was given IV opioids alone then transitioned to oral opioids. In this paper as with the paper by Cohen, readmissions were not calculated into cost-of-care. This is another example of design and reporting bias, as more subjects needed readmission in the multimodal Exparel group than in the Control group. By not including and reporting the costs associated with readmissions the authors are only reporting those data that are favorable to the Exparel group, masking the true costs of using this formulation.

Independent standardized criteria for discharge, is not evident in either of these papers leaving the determination of discharge to the investigator who was aware of the treatment assignments.

Eleven (11) studies comparing the two formulations of bupivacaine (liposomal encapsulated and plain unencapsulated, have not shown a clinical benefit for the use of the liposomal formulation. Rather, the studies have illustrated clinical equivalence between the two formulations. These studies were conducted in hemorrhoidectomy, bunionectomy, hysterectomy, colectomy, mammoplasty, and total knee replacement. The review publication authored by Bergese [10], lists 8 of these studies conducted by Pacira using unencapsulated bupivacaine as a control. Of these, 7 failed to show a difference between the two groups. In the single study that did show a difference only the highest dose of Exparel showed an effect against a less than effective non-equivalent dose of unencapsulated bupivacaine.

A recent publication by Bagsby [11] broadens the evidence that this new formulation performs no differently than an unencapsulated form of local anesthetic as used in a multimodal pain management program. This well controlled study compared the use of the liposomal bupivacaine product Exparel, to an unencapsulated form of ropivacaine as a periarticular injection in 150 consecutive patients. Treatments in both groups were identical (all procedures, postoperative care, injection techniques). Their data revealed equivalence for the first 24 hours between the two groups with the unencapsulated ropivacaine group illustrating significantly lower pain scores from 24 hours post-operative through discharge. The authors concluded, "This study found no benefit to intra-articular injection of liposomal bupivacaine, with the possible negative effect of increased pain throughout the remaining hospital course after the initial 24 hours pain control after TKA with a multimodal pain management protocol is not improved with the addition of liposomal bupivacaine compared to a traditional injection of ropivacaine and epinephrine. In addition, the additional cost of liposomal bupivacaine does not appear to be warranted over the less expensive ropivacaine injection."

\section{Discussion}

Given these observations, the primary question left is what is the benefit of this liposomal formulation of bupivacaine over the currently marketed unencapsulated formulation of bupivacaine or other local anesthetics? Promotional (advertising) pieces from the manufacturer claim that the product provides pain relief and opioid reduction for 72 hours postoperatively. From the review of the data by FDA $[2,3]$ the span of pain relief is less than 24 hours and through further analysis of the data it is closer to 12 hours. This then brings the question, is it different that plain unencapsulated bupivacaine in efficacy and safety? From the data that is published the two formulations are clinically equivalent. Where they differ is in the cost of the drugs. Liposome bupivacaine in nearly 247 times the cost of plain bupivacaine [12]. Because liposomal bupivacaine must be used in a multimodal fashion, and the overall cost of delivering pain management for 12 hours, the utility of this drug seems quite limiting.

Evidence presented in clinical trials must be held to the highest standards utilizing efforts to prevent and reduce bias in order to allow clinicians to determine which therapies may improve the quality of care for our patients. The use of the peer review process may reduce some of the inconsistencies in reporting of data in the literature however, the end users of new products also need to take responsibility for revealing bias and inconsistencies in the data reported through peer to peer communication. The evidence supporting the use of the new drug Exparel is unfortunately lacking and there are still more questions than reassuring answers regarding the analgesic efficacy and cost effectiveness of the liposomal bupivacaine formulation.

\section{References}

1. Sackett DL, Rosenberg WM, Gray JA, Haynes RB, Richardson WS (1996) Evidence based medicine: what it is and what it isn't. See comment in PubMed Commons below BMJ 312: 71-72.

2. Exparel package insert

3. Simone A (2011) Center for Drug Evaluation and Research, Clinical Review NDA 022-496 Exparel (bupivacaine)

4. $\mathrm{Li} \mathrm{Z}, \mathrm{Xu}$ Y (2010) NDA 022-496 Clinical Pharmacology and Biopharmaceutics Review.

5. Gorfine SR, Onel E, Patou G, Krivokapic ZV (2011) Bupivacaine extended-release liposome injection for prolonged postsurgical analgesia in patients undergoing hemorrhoidectomy: a multicenter, randomized, double-blind, placebo-controlled trial. See comment in PubMed Commons below Dis Colon Rectum 54: 1552-1559.

6. Golf M, Daniels SE, Onel E (2011) A phase 3, randomized, placebocontrolled trial of DepoFoam ${ }^{\oplus}$ bupivacaine (extended-release bupivacaine local analgesic) in bunionectomy. See comment in PubMed Commons below Adv Ther 28: 776-788.

7. Petullo D, Price D (2011) Statistical Review and Evaluation, Clinical Studies NDA 22-496 Exparel.

8. Marcet JE, Nfonsam VN, Larach S (2013) An extended paIn relief trial utilizing the infiltration of a long-acting Multivesicular liPosome foRmulation Of bupiVacaine, EXPAREL (IMPROVE): a Phase IV health economic trial in adult patients undergoing ileostomy reversal. See comment in PubMed Commons below J Pain Res 6: 549-555.

9. Cohen SM (2012) Extended pain relief trial utilizing infiltration of Exparel $\left({ }^{*}\right)$, a long-acting multivesicular liposome formulation of bupivacaine: a Phase IV health economic trial in adult patients undergoing open colectomy. See comment in PubMed Commons below J Pain Res 5: 567-572.

10. Bergese SD, Ramamoorthy S, Patou G, Bramlett K, Gorfine SR, et al. (2012) Efficacy profile of liposome bupivacaine, a novel formulation of 
Citation: Hadley RM, Dine AP (2014) Where is the Evidence? A Critical Review of Bias in the Reporting of Clinical Data for Exparel: A Liposomal Bupivacaine Formulation. J Clinic Res Bioeth 5: 189. doi:10.4172/2155-9627.1000189

Page 5 of 5

bupivacaine for postsurgical analgesia. See comment in PubMed Commons below J Pain Res 5: 107-116.

11. Bagsby DT, Ireland PH, Meneghini RM (2014) Liposomal Bupivacaine Versus Traditional Periarticular Injection for Pain Control After Total Knee Arthroplasty. See comment in PubMed Commons below J Arthroplasty
12. Nadeau M, Saraswat A, Vasko A, Vasko S (2013) Bupivacaine versus Depofoam Bupivacaine in Breast Augmentation: A prospective randomized double blind study, Presented at the Ohio Society of Plastic Surgeons annual meeting, \#191. 